\title{
Photothermal Response of Polyhydroxy Fullerenes
}

\author{
Alan Chen', Stephen R. Grobmyer,'s and Vijay B. Krishna ${ }^{*},+$ \\ †Department of Biomedical Engineering, Lerner Research Institute, *Surgical Oncology, Digestive Disease \\ Institute, Cleveland Clinic, Cleveland, OH 44195
}

*Corresponding Author: Vijay Krishna (krishnv2@ccf.org)

${ }^{\S}$ Current Address: Oncology Institute, Cleveland Clinic Abu Dhabi, Abu Dhabi, United Arab Emirates 


\section{Supplementary Figures}
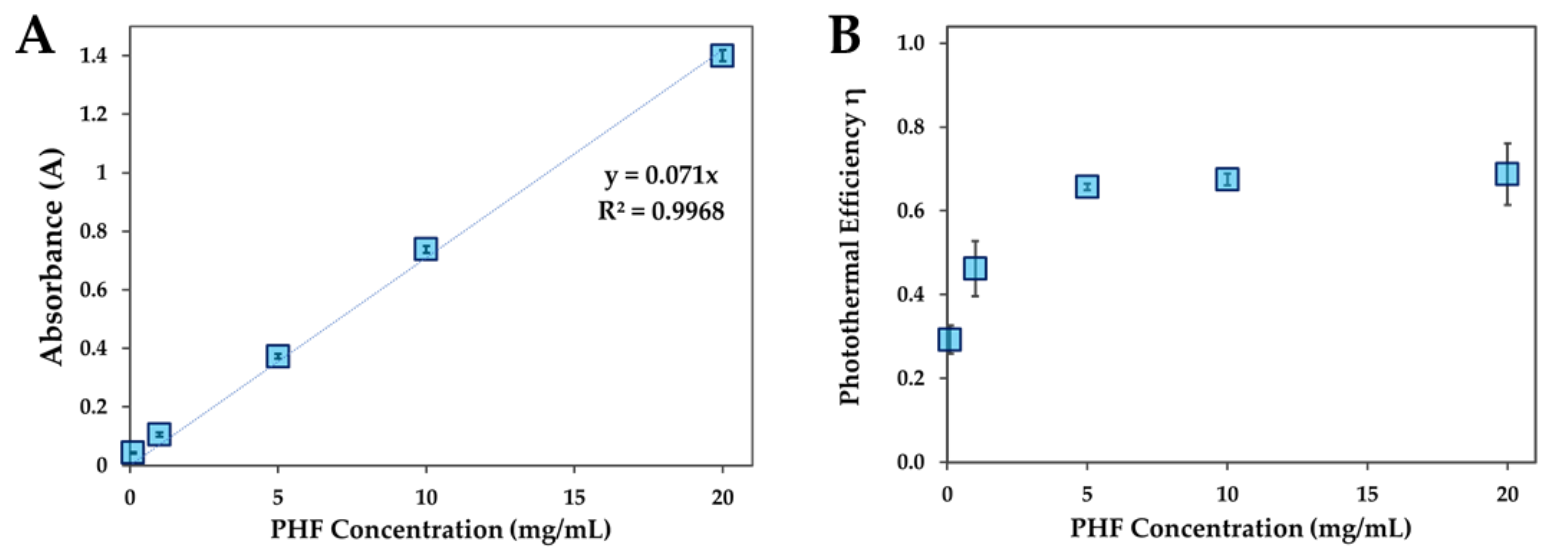

Figure S1: A) Ground state absorption of PHF as a function of concentration at $785 \mathrm{~nm}$. PHF has linear absorption in tested concentrations. PHF's extinction coefficient at $785 \mathrm{~nm}$ is $0.071 \mathrm{~mL} /(\mathrm{mg} \mathrm{cm})$. B) Photothermal conversion efficiency as a function of PHF concentration. The photothermal efficiency increases linearly at low concentrations and plateaus at higher concentrations. All experiments were conducted in triplicate $(\mathrm{n}=3)$ and error bars represent standard deviations. 

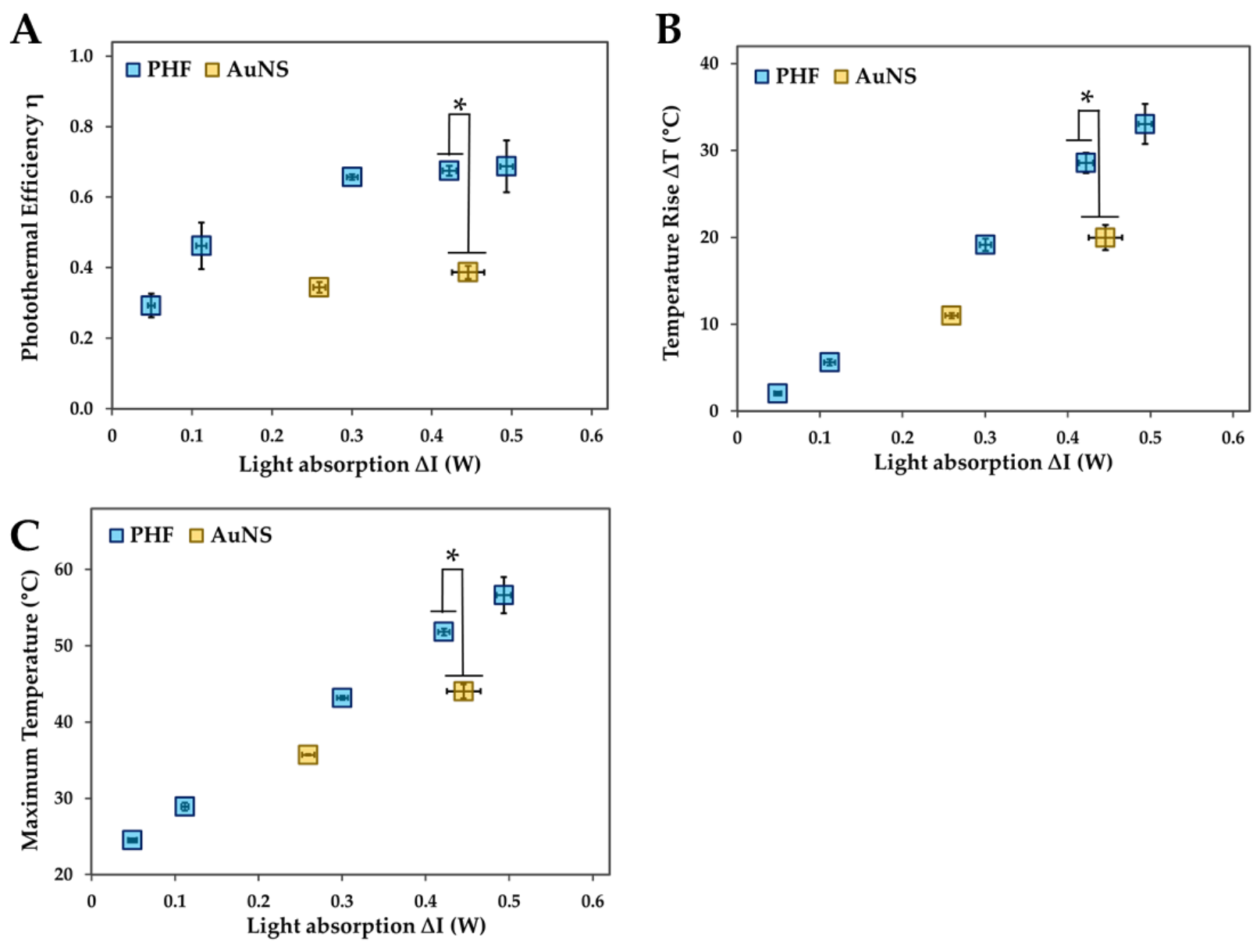

Figure S2: Effect of light absorption $(\Delta I)$ on A) photothermal efficiency $(\eta), \mathrm{B})$ temperature rise $(\Delta T)$ and C) maximum temperature. At similar light absorptions, PHF has significantly $(p>0.01)$ higher photothermal efficiency than gold nanoshells (AuNS). All experiments were conducted in triplicate $(n=3)$ and error bars represent standard deviations. ${ }^{*}$ indicates significance at $\alpha=0.01$. 
Table S1

\begin{tabular}{|c|c|c|c|c|c|c|c|c|c|}
\hline $\begin{array}{c}\text { Photothermal } \\
\text { Agent }\end{array}$ & $\begin{array}{c}\text { Laser } \\
\text { Wavelength }\end{array}$ & $\begin{array}{l}\text { Cuvette } \\
\text { material }\end{array}$ & $t(\mathrm{sec})$ & $\begin{array}{c}I_{0} \\
\left(\mathrm{~W} / \mathrm{cm}^{2}\right)\end{array}$ & $\begin{array}{l}\text { Conc of } \\
\text { NPs }\end{array}$ & $\begin{array}{c}\text { Converted } \\
\text { Conc. } \\
\text { (mg/mL) }\end{array}$ & $\begin{array}{c}\eta \\
\text { Calculation } \\
\text { Method }\end{array}$ & $\eta(\%)$ & References \\
\hline PHF & $783 \mathrm{~nm}$ & PMMA & 1800 (plateau) & 0.5 & $10 \mathrm{mg} / \mathrm{mL}$ & 10 & optimization & 69 & This work \\
\hline AuNS & $784 \mathrm{~nm}$ & PMMA & 1800 (plateau) & 0.5 & $31.2 \mu \mathrm{g} / \mathrm{mL}$ & 0.0312 & optimization & 39 & This work \\
\hline AuNS & $815 \mathrm{~nm}$ & PMMA & 2400 (plateau) & 1.50 & $3 \mathrm{pM}$ & 0.0312 & time constant & 30 & 1 \\
\hline $\operatorname{AuNR}(8 \mathrm{~nm})$ & $809 \mathrm{~nm}$ & Quartz & 1800 (plateau) & 1.72 & $0.24 \mathrm{nM}$ & 0.57 & time constant & 95 & 2 \\
\hline AuNR (20 nm) & $809 \mathrm{~nm}$ & Quartz & 1800 (plateau) & 1.72 & $0.24 \mathrm{nM}$ & 0.57 & time constant & 90 & 2 \\
\hline AuNR (27 nm) & $980 \mathrm{~nm}$ & Quartz & 600 (plateau) & 0.51 & $40 \mu \mathrm{g} / \mathrm{mL}$ & 0.04 & time constant & 23.7 & 3 \\
\hline AuNR (30 nm) & $980 \mathrm{~nm}$ & Quartz & 360 (plateau) & 1 & $50 \mu \mathrm{g} / \mathrm{mL}$ & 0.05 & time constant & 78.8 & 4 \\
\hline AuNR (36 nm) & $809 \mathrm{~nm}$ & Quartz & 1800 (plateau) & 1.72 & $30 \mu \mathrm{g} / \mathrm{mL}$ & 0.03 & time constant & 95 & 5 \\
\hline AuNR (40 nm) & $808 \mathrm{~nm}$ & Quartz & $\begin{array}{l}300 \text { (no } \\
\text { plateau) }\end{array}$ & 0.1 & $\begin{array}{l}\text { Not } \\
\text { provided }\end{array}$ & & time constant & $27-33$ & 6 \\
\hline AuNR (45 nm) & $815 \mathrm{~nm}$ & PMMA & 3600 (plateau) & 1.50 & $100 \mathrm{pM}$ & 0.24 & time constant & 55 & 1 \\
\hline AuNR (50 nm) & $809 \mathrm{~nm}$ & Quartz & 1800 (plateau) & 1.72 & $0.24 \mathrm{nM}$ & 0.57 & time constant & 50 & 2 \\
\hline AuNR (60 nm) & $809 \mathrm{~nm}$ & Quartz & 1800 (plateau) & 1.72 & $30 \mu \mathrm{g} / \mathrm{mL}$ & 0.03 & time constant & 89 & 5 \\
\hline AuNR (60 nm) & $808 \mathrm{~nm}$ & & $\begin{array}{l}600 \text { (no } \\
\text { plateau) }\end{array}$ & 0.6 & $25 \mu \mathrm{g} / \mathrm{mL}$ & 0.025 & time constant & 39 & 7 \\
\hline AuNR (60 nm) & $808 \mathrm{~nm}$ & PP & 300 (plateau) & 0.2 & $100 \mu \mathrm{g} / \mathrm{mL}$ & 0.1 & time constant & 36.2 & 8 \\
\hline AuNR (70 nm) & $809 \mathrm{~nm}$ & Quartz & 1800 (plateau) & 1.72 & $30 \mu \mathrm{g} / \mathrm{mL}$ & 0.03 & time constant & 82 & 5 \\
\hline AuNP (5 nm) & $532 \mathrm{~nm}$ & Quartz & 1200 (plateau) & 0.25 & $0.2 \mathrm{mM}$ & & time constant & 80 & 9 \\
\hline AuNP (10 nm) & $808 \mathrm{~nm}$ & Quartz & $\begin{array}{l}300 \text { (no } \\
\text { plateau) }\end{array}$ & 0.1 & $\begin{array}{l}\text { Not } \\
\text { provided }\end{array}$ & & time constant & 9 & 6 \\
\hline
\end{tabular}




\begin{tabular}{|c|c|c|c|c|c|c|c|c|c|}
\hline AuNP (15 nm) & $532 \mathrm{~nm}$ & Quartz & 1200 (plateau) & 0.25 & $0.2 \mathrm{mM}$ & & time constant & 78 & 9 \\
\hline AuNP (20 nm) & $514 \mathrm{~nm}$ & Quartz & 320 (plateau) & 0.17 & $920 \mu \mathrm{g} / \mathrm{mL}$ & 0.92 & time constant & 8 & 10 \\
\hline AuNP (50 nm) & $532 \mathrm{~nm}$ & Quartz & 1200 (plateau) & 0.25 & $0.2 \mathrm{mM}$ & & time constant & 65 & 9 \\
\hline AuNP (127 nm) & $809 \mathrm{~nm}$ & Quartz & 1800 (plateau) & 1.72 & $33 \mu \mathrm{g} / \mathrm{mL}$ & 0.033 & time constant & 60 & 5 \\
\hline AuNP (154 nm) & $809 \mathrm{~nm}$ & Quartz & 1800 (plateau) & 1.72 & $33 \mu \mathrm{g} / \mathrm{mL}$ & 0.033 & time constant & 45 & 5 \\
\hline $\begin{array}{l}\text { Au Nano Cube (10 } \\
\mathrm{nm})\end{array}$ & $808 \mathrm{~nm}$ & Quartz & $\begin{array}{l}300 \text { (no } \\
\text { plateau) }\end{array}$ & 0.1 & $\begin{array}{l}\text { Not } \\
\text { provided }\end{array}$ & & time constant & 15 & 6 \\
\hline AuNR-CuS (60 nm) & $808 \mathrm{~nm}$ & & $\begin{array}{l}600 \text { (no } \\
\text { plateau) }\end{array}$ & 0.6 & $25 \mu \mathrm{g} / \mathrm{mL}$ & 0.025 & time constant & 62 & 7 \\
\hline AuNP-Co $(50 \mathrm{~nm})$ & $790 \mathrm{~nm}$ & & 1800 (plateau) & 1.0 & $0.2 \mathrm{mg} / \mathrm{mL}$ & 0.2 & time constant & 99 & 11 \\
\hline Au/Mo-ICG (110 nm) & $808 \mathrm{~nm}$ & PP & 300 (plateau) & 0.2 & $100 \mu \mathrm{g} / \mathrm{mL}$ & 0.1 & time constant & 68.8 & 8 \\
\hline $\begin{array}{l}\mathrm{Au}-\mathrm{Pt} \\
\text { Nanoraspberries }\end{array}$ & $650 \mathrm{~nm}$ & Quartz & $\begin{array}{l}300 \text { (no } \\
\text { plateau) }\end{array}$ & 0.1 & \begin{tabular}{|l|} 
Not \\
provided \\
\end{tabular} & & time constant & 61 & 12 \\
\hline $\begin{array}{l}\mathrm{Au}-\mathrm{Pt} \\
\text { Nanoraspberries }\end{array}$ & $808 \mathrm{~nm}$ & Quartz & $\begin{array}{l}300 \text { (no } \\
\text { plateau) }\end{array}$ & 0.1 & $\begin{array}{l}\text { Not } \\
\text { provided }\end{array}$ & & time constant & 52 & 12 \\
\hline $\begin{array}{l}\text { AgI-AgS2 } \\
\text { Nanostructure }\end{array}$ & 808 nm & Quartz & $\begin{array}{l}3000 \text { (no } \\
\text { plateau) }\end{array}$ & 0.42 & $0.01 \% \mathrm{wt}$ & 10 & time constant & 33.4 & 13 \\
\hline NiS2, Mesoporous & $808 \mathrm{~nm}$ & Quartz & $\begin{array}{l}600 \text { (no } \\
\text { plateau) }\end{array}$ & 2 & $20 \mu \mathrm{g} / \mathrm{mL}$ & 0.02 & time constant & 44.6 & 14 \\
\hline Liquid Metal NR & $808 \mathrm{~nm}$ & PS & $\begin{array}{l}250 \text { (no } \\
\text { plateau) }\end{array}$ & 3.5 & $800 \mathrm{mg} / \mathrm{L}$ & 0.8 & time constant & 25.3 & 15 \\
\hline GaNS & $808 \mathrm{~nm}$ & PS & $\begin{array}{l}250 \text { (no } \\
\text { plateau) }\end{array}$ & 3.5 & $800 \mathrm{mg} / \mathrm{L}$ & 0.8 & time constant & 25.3 & 15 \\
\hline GaNR & $808 \mathrm{~nm}$ & PS & $\begin{array}{l}250 \text { (no } \\
\text { plateau) }\end{array}$ & 3.5 & $800 \mathrm{mg} / \mathrm{L}$ & 0.8 & time constant & 32.7 & 15 \\
\hline $\mathrm{Cu}$ Nanocluster & $980 \mathrm{~nm}$ & Quartz & 600 (plateau) & 0.51 & $40 \mu \mathrm{g} / \mathrm{mL}$ & 0.04 & time constant & 25.7 & 3 \\
\hline Cu Nanocluster & $800 \mathrm{~nm}$ & Quartz & $\begin{array}{l}300 \text { (no } \\
\text { plateau) }\end{array}$ & 2 & $1 \mathrm{mg} / \mathrm{mL}$ & 1 & time constant & 22 & 16 \\
\hline
\end{tabular}




\begin{tabular}{|c|c|c|c|c|c|c|c|c|c|}
\hline $\begin{array}{l}\text { Pt Nanoparticles (2 } \\
\mathrm{nm})\end{array}$ & $808 \mathrm{~nm}$ & Quartz & $\begin{array}{l}300 \text { (no } \\
\text { plateau) }\end{array}$ & 0.1 & & & time constant & 33 & 17 \\
\hline $\begin{array}{l}\text { Pt Nanoparticles (80 } \\
\mathrm{nm})\end{array}$ & $808 \mathrm{~nm}$ & Quartz & $\begin{array}{l}300(\text { no } \\
\text { plateau) }\end{array}$ & 0.1 & & & time constant & 15 & 17 \\
\hline ICG & $808 \mathrm{~nm}$ & PS & $\begin{array}{l}180 \text { (no } \\
\text { plateau) }\end{array}$ & 1.1 & $5 \mu \mathrm{g} / \mathrm{mL}$ & 0.005 & time constant & 3.37 & 18 \\
\hline Cyanine Micelles & $808 \mathrm{~nm}$ & & $\begin{array}{l}600 \text { (no } \\
\text { plateau) }\end{array}$ & 0.7 & $5 \mathrm{ug} / \mathrm{mL}$ & 0.005 & & 47 & 19 \\
\hline Organic Drug & $635 \mathrm{~nm}$ & & $\begin{array}{l}300 \text { (no } \\
\text { plateau) }\end{array}$ & 0.5 & $0.5 \mathrm{mM}$ & & time constant & 37 & 20 \\
\hline Carbon Nanospheres & $808 \mathrm{~nm}$ & PS & $\begin{array}{l}600 \text { (no } \\
\text { plateau) }\end{array}$ & 1.5 & $100 \mu \mathrm{g} / \mathrm{mL}$ & 0.1 & time constant & 35.7 & 21 \\
\hline
\end{tabular}

$\boldsymbol{t}=$ Laser irradiation time; $\boldsymbol{I}_{0}=$ Incident laser power $\boldsymbol{\eta}=$ Photothermal conversion efficiency 


\section{$\underline{\text { References }}$}

1. Cole, J. R.; Mirin, N. A.; Knight, M. W.; Goodrich, G. P.; Halas, N. J., Photothermal Efficiencies of Nanoshells and Nanorods for Clinical Therapeutic Applications. J. Phys. Chem. C 2009, 113 (28), 12090-12094.

2. Chen, H.; Shao, L.; Ming, T.; Sun, Z.; Zhao, C.; Yang, B.; Wang, J., Understanding the photothermal conversion efficiency of gold nanocrystals. Small 2010, 6 (20), 2272-80.

3. Tian, Q.; Jiang, F.; Zou, R.; Liu, Q.; Chen, Z.; Zhu, M.; Yang, S.; Wang, J.; Wang, J.; Hu, J., Hydrophilic Cu9S5 Nanocrystals: A Photothermal Agent with a 25.7\% Heat Conversion Efficiency for Photothermal Ablation of Cancer Cells in Vivo. ACS Nano 2011, 5 (12), 9761-9771.

4. $\quad$ Bi, C.; Chen, J.; Chen, Y.; Song, Y.; Li, A.; Li, S.; Mao, Z.; Gao, C.; Wang, D.; Möhwald, H.; Xia, H., Realizing a Record Photothermal Conversion Efficiency of Spiky Gold Nanoparticles in the Second Near-Infrared Window by Structure-Based Rational Design. Chem. Mater. 2018, 30 (8), 2709-2718.

5. Jiang, K.; Smith, D. A.; Pinchuk, A., Size-Dependent Photothermal Conversion Efficiencies of Plasmonically Heated Gold Nanoparticles. J. Phys. Chem. C 2013, 117 (51), 27073-27080.

6. Depciuch, J.; Stec, M.; Kandler, M.; Baran, J.; Parlinska-Wojtan, M., From spherical to bone-shaped gold nanoparticles-Time factor in the formation of Au NPs, their optical and photothermal properties. Photodiagn. Photodyn. Ther. 2020, 101670.

7. Leng, C.; Zhang, X.; Xu, F.; Yuan, Y.; Pei, H.; Sun, Z.; Li, L.; Bao, Z., Engineering Gold Nanorod-Copper Sulfide Heterostructures with Enhanced Photothermal Conversion Efficiency and Photostability. Small 2018, 14 (12), e1703077.

8. Younis, M. R.; Wang, C.; An, R.; Wang, S.; Younis, M. A.; Li, Z.-Q.; Wang, Y.; Ihsan, A.; Ye, D.; Xia, X.-H., Low Power Single Laser Activated Synergistic Cancer Phototherapy Using Photosensitizer Functionalized Dual Plasmonic Photothermal Nanoagents. ACS Nano 2019, 13 (2), 2544-2557.

9. Jiang, R.; Cheng, S.; Shao, L.; Ruan, Q.; Wang, J., Mass-Based Photothermal Comparison Among Gold Nanocrystals, PbS Nanocrystals, Organic Dyes, and Carbon Black. J. Phys. Chem. C 2013, 117 (17), 8909-8915.

10. Roper, D. K.; Ahn, W.; Hoepfner, M., Microscale Heat Transfer Transduced by Surface Plasmon Resonant Gold Nanoparticles. J. Phys. Chem. C 2007, 111 (9), 3636-3641.

11. Lindley, S. A.; Zhang, J. Z., Bumpy Hollow Gold Nanospheres for Theranostic Applications: Effect of Surface Morphology on Photothermal Conversion Efficiency. ACS Appl. Nano Mater. 2019, 2 (2), 1072-1081.

12. Depciuch, J.; Stec, M.; Klebowski, B.; Baran, J.; Parlinska-Wojtan, M., Platinum-gold nanoraspberries as effective photosensitizer in anticancer photothermal therapy. J. Nanobiotechnol. 2019, 17 (1), 107.

13. Zeng, W.; Suo, L.; Zhang, C.; Wu, D.; Zhu, H., AgI-Ag2S heterostructures for photothermal conversion and solar energy harvesting. J. Taiwan Inst. Chem. Eng. 2019, 95, 273-280. 
14. He, G.; Ma, Y.; Zhou, H.; Sun, S.; Wang, X.; Qian, H.; Xu, Y.; Miao, Z.; Zha, Z., Mesoporous NiS2 nanospheres as a hydrophobic anticancer drug delivery vehicle for synergistic photothermal-chemotherapy. J. Mater. Chem. B 2019, 7 (1), $143-149$.

15. Sun, X.; Sun, M.; Liu, M.; Yuan, B.; Gao, W.; Rao, W.; Liu, J., Shape tunable gallium nanorods mediated tumor enhanced ablation through near-infrared photothermal therapy. Nanoscale 2019, 11 (6), 2655-2667.

16. Hessel, C. M.; Pattani, V. P.; Rasch, M.; Panthani, M. G.; Koo, B.; Tunnell, J. W.; Korgel, B. A., Copper Selenide Nanocrystals for Photothermal Therapy. Nano Lett. 2011, 11 (6), 2560-2566.

17. Depciuch, J.; Stec, M.; Klebowski, B.; Maximenko, A.; Drzymala, E.; Baran, J.; Parlinska-Wojtan, M., Size effect of platinum nanoparticles in simulated anticancer photothermal therapy. Photodiagn. Photodyn. Ther. 2020, $29,101594$.

18. Yoon, H.-J.; Lee, H.-S.; Lim, J.-Y.; Park, J.-H., Liposomal Indocyanine Green for Enhanced Photothermal Therapy. ACS Appl. Mater. Interfaces 2017, 9 (7), 5683-5691.

19. Li, L.; Yang, Q.; Shi, L.; Zheng, N.; Li, Z.; Li, K.; Qiao, S.; Jia, T.; Sun, T.; Wang, Y., Novel phthalocyanine-based polymeric micelles with high near-infrared photothermal conversion efficiency under $808 \mathrm{~nm}$ laser irradiation for in vivo cancer therapy. J. Mater. Chem. B 2019, 7 (14), 2247-2251.

20. Wang, H.; Chang, J.; Shi, M.; Pan, W.; Li, N.; Tang, B., A Dual-Targeted Organic Photothermal Agent for Enhanced Photothermal Therapy. Angew. Chem., Int. Ed. Engl. 2019, 58 (4), 1057-1061.

21. Weng, Y.; Guan, S.; Wang, L.; Qu, X.; Zhou, S., Hollow carbon nanospheres derived from biomass by-product okara for imagingguided photothermal therapy of cancers. J. Mater. Chem. B 2019, 7 (11), 1920-1925. 